12

\title{
Электронная структура вариантов составов скандатных и оксидно-никелевых катодов СВЧ приборов
}

\author{
(С) В.И. Капустин, ${ }^{1}$ И.П. Ли, ${ }^{2}$ А.С. Серпичев, ${ }^{1}$ А.В. Шуманов, ${ }^{2}$ Н.Е. Кожевникова ${ }^{2}$ \\ ${ }^{1}$ МИРЭА - Российский технологический университет, \\ 119454 Москва, Россия \\ ${ }^{2} \mathrm{AO}$, „лутон“, \\ 105120 Москва, Россия \\ e-mail: kapustin@mirea.ru
}

Поступило в Редакцию 9 июля 2021 г.

В окончательной редакции 28 ноября 2021 г.

Принято к публикации 8 декабря 2021 г.

\begin{abstract}
Методами электронной спектроскопии для химического анализа и спектроскопии характеристических потерь энергии электронов исследована электронная структура кристаллитов оксида бария, легированного другими химическими элементами, в том числе скандием из скандийсодержащих фаз. Сформулированы физические и физико-химические условия, выполнение которых позволяет сформировать электронную структуру скандатного катода с высоким уровнем термоэлектронной эмиссии: достижение минимума отношения поверхностной объемной концентрации кислородных вакансий и максимума расстояния между верхом валентной зоны и уровнем Ферми.
\end{abstract}

Ключевые слова: СВЧ приборы, катоды, термоэлектронная эмиссия, работа выхода, электронная структура, электронная спектроскопия.

DOI: $10.21883 / J T F .2022 .03 .52143 .211-21$

\section{Введение}

Начиная с 90-х годов прошлого века научные лаборатории университетов и промышленных предприятий во всех развитых странах проводят исследования, направленные на создание так называемых скандатных катодов, т.е. металлопористых катодов, содержащих в своем составе скандий. По результатам исследований установлено, что такой катод может обеспечить получение плотности тока термоэлектронной эмиссии до $100 \mathrm{~A} / \mathrm{cm}^{2}$, а в перспективе и до $400 \mathrm{~A} / \mathrm{cm}^{2}$ [1-6]. Это открывает возможность создания принципиально новых типов электровакуумных СВЧ приборов. В то же время полученные результаты свидетельствуют о плохой технологической воспроизводимости эмиссионных свойств скандатных катодов, поэтому до настоящего времени никакие промышленные предприятия не освоили серийный выпуск СВЧ приборов с катодами такого типа.

На рис. 1 приведена схема возможных путей создания катодного материала с заданными эмиссионными свойствами. В соответствии с данной схемой научную методологию большинства опубликованных работ можно отнести к исследованию направления (взаимосвязи) 1 данной схемы, когда апробируют различные технологические приемы изготовления катода и измеряют набор эмиссионных свойств продукта технологий термоэмиссионные и вторично-эмиссионные свойства, стабильность и долговечность свойств, допустимый температурный интервал работы катодного материала.

Так как эмиссионные свойства катодного материала определяются электронной структурой эмиссионно- активной фазы материала (кристаллитов $\mathrm{BaO}$ ), процесс формирования этой фазы на этапе активирования катода определяется исходным фазовым составом материала, исходной микроструктурой материала, микропримесями, которые внедряются в состав активной фазы из других фаз на этапе активирования и работы катода. Поэтому „Проектирование“ эмиссионных свойств катода с заданными эмиссионными свойствами, на наш взгляд, целесообразно вести на основе результатов исследований направлений $2-5$ на рис. 1. При установлении закономерностей направлений 2-5 выбор технологий по направлениям 6-8, обеспечивающих формирование необходимых

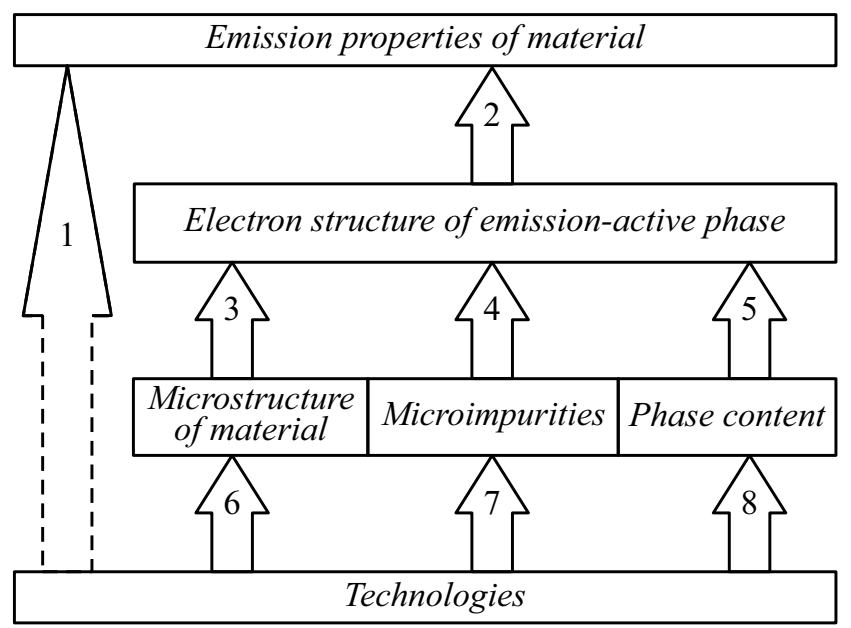

Рис. 1. Схема разработки катодного материала с заданными эмиссионными свойствами. 
исходных фазового состава и микроструктуры материала, микролегирование эмиссионно-активной фазы требуемыми микропримесями, будет предопределен почти автоматически на основе известного набора возможных технологических операций.

Впервые целесообразность подхода к созданию катодных материалов на основе изучения направлений 2,3 и 5 была сформулирована в работах [7,8], а важность изучения направления 4 была теоретически и экспериментально установлена в работах [9-13], причем в работе [10] был впервые экспериментально установлен синергетических эффект влияния микропримесей кальция и стронция на электронную структуру кристаллитов оксида бария. Полученные экспериментальные и теоретические результаты по различным типам катодных материалов были обобщены в монографии [14].

Вместе с тем возможное совместное (синергетическое) проявление влияния микропримесей в кристаллитах $\mathrm{BaO}$ на его электронную структуру оказалось недостаточно изученным, а также недостаточно исследованным оказалось влияние фазового состояния, в частности, компонентов, содержащих скандий, на электронную структуру эмиссионно-активной фазы катодного материала и синергетику ее микролегирования микропримесями. Поэтому целью настоящей работы являлось исследование электронной структуры материалов и поиск эффектов, в том числе синергетических, влияния микропримесей в $\mathrm{BaO}$ на два параметра, определяющих величину его работы выхода: достижение минимума отношения поверхностной и объемной концентраций кислородных вакансий и максимума расстояния между верхом валентной зоны и уровнем Ферми.

\section{1. Экспериментальные образцы и методики исследований}

Для исследования влияния микролегирования на электронную структуру кристаллитов $\mathrm{BaO}$, прежде всего на объемную и поверхностную концентрацию кислородных вакансий в кристаллитах $\mathrm{BaO}$, а также для исследования возможного синергетического эффекта двойного легирования, были изготовлены две группы экспериментальных образцов.

В первой группе образцы материалов в виде таблеток диаметром $7 \mathrm{~mm}$ и толщиной $1 \mathrm{~mm}$ получали спеканием и последующим прессованием таблеток из смеси порошка карбоната бария и порошков дополнительных компонентов в количестве 10\% (весовых) - оксида иттрия, рения, палладия, карбоната стронция. Спекание порошков проводили в вакууме при температуре $1200^{\circ} \mathrm{C}$ в течение $2 \mathrm{~h}$ на никелевой пластине. Так как при указанной температуре никель обладает заметной летучестью, в результате спекания в образцах материалов формировались кристаллиты $\mathrm{BaO}$, содержащие кислородные вакансии, кристаллиты $\mathrm{BaO}$, содержащие кислородные вакансии и легированные атомами дополнительного компонента (иттрия, рения, палладия, стронция), а также кристаллиты $\mathrm{BaO}$, содержащие кислородные вакансии и легированные атомами дополнительного компонента и атомами никеля.

Во второй группе образцы материалов в виде таблеток диаметром $7 \mathrm{~mm}$ и толщиной $1 \mathrm{~mm}$ получали спеканием и последующим прессованием таблеток из смеси порошка вольфрама (90 весовых \%), предварительно синтезированного и размолотого в порошок алюмината бариякальция состава $2.5 \mathrm{BaO} \cdot 0.4 \mathrm{CaO} \cdot \mathrm{Al}_{2} \mathrm{O}_{3}$ и дополнительных компонентов в виде порошков интерметаллида $\mathrm{Re}_{2} \mathrm{Sc}$, порошка сплава $80 \% \mathrm{~W}+20 \% \mathrm{Re}$, порошка гидрида скандия $\mathrm{ScH}_{2}$. Спекание порошков также проводили в вакууме при температуре $1200^{\circ} \mathrm{C}$ в течение $2 \mathrm{~h}$, но на вольфрамовой пластине. В результате спекания в образцах материалов формировались кристаллиты $\mathrm{BaO}$, содержащие кислородные вакансии, а также кристаллиты $\mathrm{BaO}$, содержащие кислородные вакансии и в зависимости от состава смесей легированные атомами вольфрама, рения, скандия и комбинацией указанных элементов.

Порошок интерметаллида $\mathrm{Re}_{2} \mathrm{Sc}$ был получен электродуговой переплавкой компонентов в среде очищенного аргона с последующим размолом в шаровой мельнице. Порошок гидрида скандия $\mathrm{ScH}_{2}$ был получен отжигом скандия в среде водорода с последующим размолом в шаровой мельнице. Порошок сплава $80 \% \mathrm{~W}+20 \% \mathrm{Re}$ был изготовлен в Научно-производственном комплексе „Передовые порошковые технологии“ (г. Томск) распылением проволоки ВР-20 (сплав 80\% $+20 \% \mathrm{Re}$ ) методом электровзрыва. Порошок сплава ВР-20 состоит из частиц диаметром $1-2 \mu \mathrm{m}$ и фракции частиц порошка субмикронных размеров.

Электронные состояния элементов в образцах материалов исследовали методом электронной спектрометрии для химического анализа (ЭСХА), при этом расшифровку спектров проводили путем разбиения пиков в спектрах ЭСХА на гауссовы пики с учетом влияния окружения атома другими элементами на сдвиги пиков атомов, которые зависят от величины электроотрицательности указанных элементов. В качестве примера на рис. 2 приведена структура $3 d_{5 / 2}$-электронного уровня бария в образце материала $90 \% \mathrm{BaCO}_{3}+10 \% \mathrm{Y}_{2} \mathrm{O}_{3}$, а на рис. $3-$

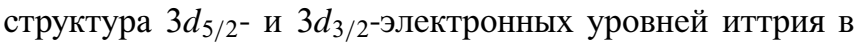
этом же образце материала. Расшифровка электронных состояний бария и иттрия в указанном образце приведена в табл. 1 (п.п. $1.1-1.4$ соответствуют пикам 1-4 на рис. 2 ) и в табл. 2 (п.п. $1-4$ соответствуют пикам 1-4 на рис. 3).

Концентрацию кислородных вакансий в образцах материалов исследовали методом спектроскопии характеристических потерь энергии электронов (СХПЭЭ) при энергии первичных электронов $1005 \mathrm{eV}$ с шагом регистрации $0.05 \mathrm{eV}$. Для повышения чувствительности 


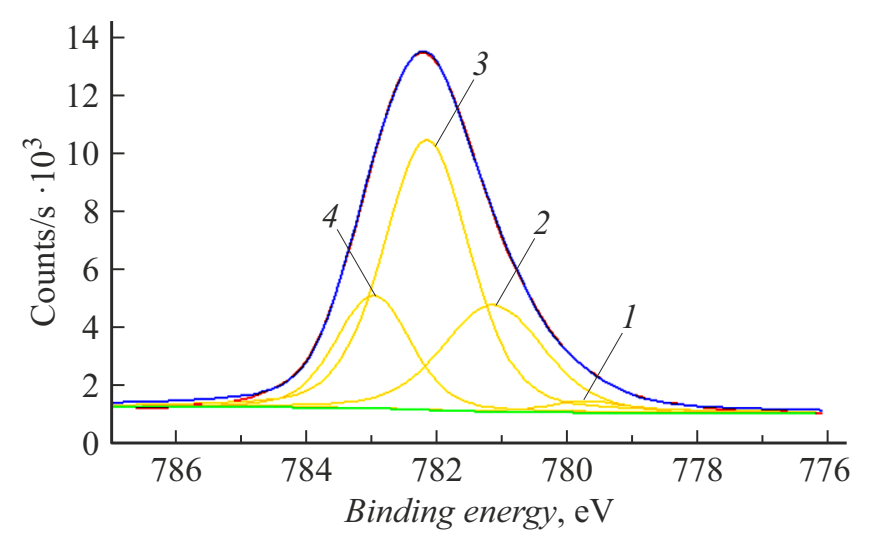

Рис. 2. Структура $3 d_{5 / 2}$-электронного уровня бария в образце материала $\mathrm{BaCO}_{3}+10 \% \mathrm{Y}_{2} \mathrm{O}_{3}$, отожженного в вакууме при $1200^{\circ} \mathrm{C}$ в течение $2 \mathrm{~h}$.

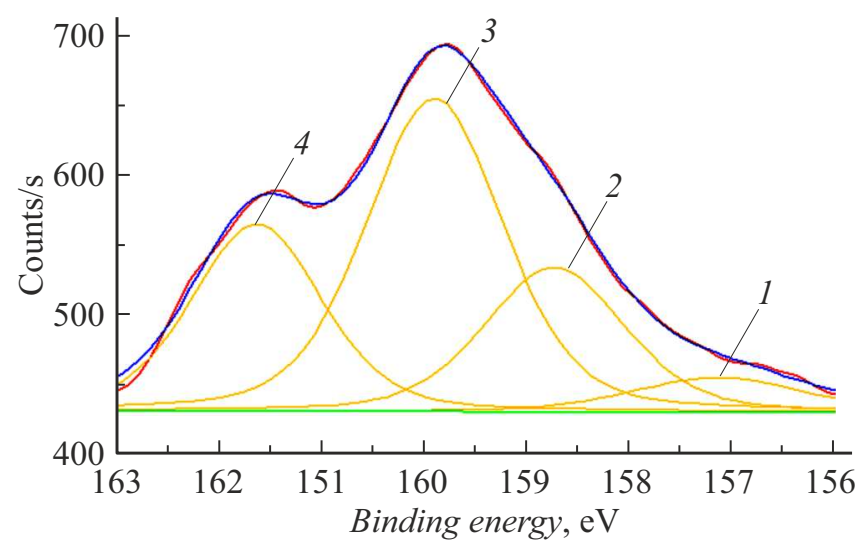

Рис. 3. Структуры $3 d_{5 / 2-}$ и $3 d_{3 / 2}$-электронных уровней иттрия в образце материала $\mathrm{BaCO}_{3}+10 \% \mathrm{Y}_{2} \mathrm{O}_{3}$, отожженного в вакууме при $1200^{\circ} \mathrm{C}$ в течение $2 \mathrm{~h}$.

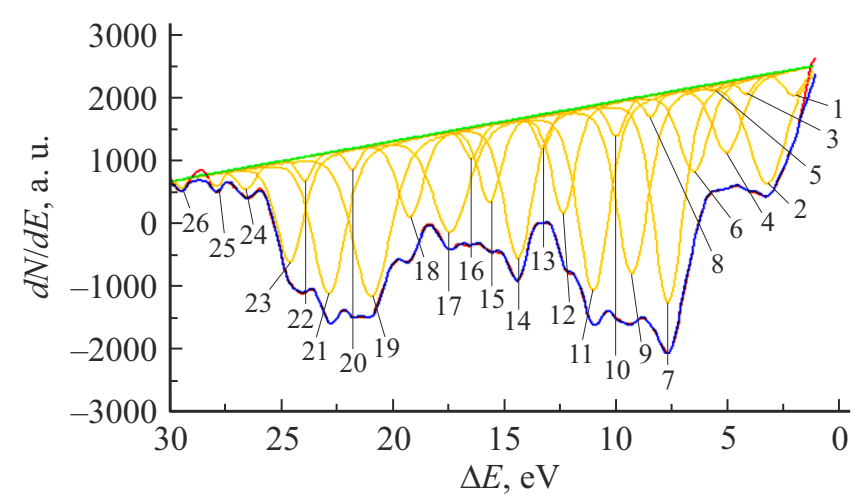

Рис. 4. Спектр характеристических потерь энергии электронов в образце материала $\mathrm{BaCO}_{3}+10 \% \mathrm{Y}_{2} \mathrm{O}_{3}$, отожженного в вакууме при $1200^{\circ} \mathrm{C}$ в течение $2 \mathrm{~h}$.

метода проводили цифровое дифференцирование спектров. В качестве примера на рис. 4 приведен дифференциальный спектр характеристических потерь энергии электронов в образце материала $90 \% \mathrm{BaCO}_{3}+10 \% \mathrm{Y}_{2} \mathrm{O}_{3}$.
Характеристические потери энергии электронов в исследованных образцах материалов обусловлены возбуждением поверхностных и объемных плазмонов в металлической фазе материалов из второй группы образцов (вольфрам), а также возбуждением поверхностных и объемных плазмонов в кристаллитах оксида бария, содержащих кислородные вакансии, которые формируют точечные дефекты донорного типа $[7,8]$, и примесные атомы. В этом случае энергии возбуждаемых поверхностных $\Delta E_{S}$ и объемных $\Delta E_{D}$ плазмонов связаны с поверхностной $N_{S}$ и объемной $N_{D}$ концентрацией кислородных вакансий соотношениями [14]:

$$
\Delta E_{D}=\sqrt{\frac{e^{* 2} \hbar^{2} N_{D}}{\varepsilon \varepsilon_{0} m^{*}}}, \quad \Delta E_{S}=\sqrt{\frac{e^{* 2} \hbar^{2} N_{S}}{2 \varepsilon \varepsilon_{0} m^{*}}},
$$

где $e^{*}$ - эффективный заряд кислородной вакансии, $m^{*}$ - эффективная масса электронов кислородных вакансий, $\hbar-$ постоянная Планка, $\varepsilon_{0}$ - диэлектрическая постоянная, $\varepsilon=3.6$ - высокочастотная диэлектрическая проницаемость оксида бария. Тогда энергия плазменных потерь электронов составит

$$
\Delta E=n_{1} \Delta E_{D}+n_{2} \Delta E_{S},
$$

где $n_{1}$ и $n_{2}$ - целые числа.

При расчете поверхностной и объемной концентраций кислородных вакансий значения эффективной массы электронов на кислородных вакансиях и эффективного заряда вакансий в чистых кристаллитах $\mathrm{BaO}$ и в кристаллитах, легированных одним типом микропримесей, брали из [12] и [14], где они были определены экспериментально методом оптического поглощения. Значения указанных параметров для кристаллитов $\mathrm{BaO}$, легированного двумя типами микропримесей, в настоящее время экспериментально не определены. Поэтому для указанного случая двойного легирования при расчетах по соотношениям (1) и (2) брали значения эффективной массы и эффективного заряда для компонента, содержащегося в материале в максимальной концентрации. Такой выбор мог приводить к определенной погрешности при расчете абсолютных значений поверхностной и объемной концентраций вакансий, но не влиял на важнейший параметр - отношение поверхностной и объемной концентраций вакансий в каждом образце.

При расчете поверхностной и объемной концентраций электронов в металлической фазе образцов материалов формально можно использовать соотношения (1) и (2), если положить для металлов $e^{*}=e, m^{*}=m, \varepsilon=1$. В качестве примера в табл. 3 и 4 приведена схема расшифровки спектра характеристических потерь образца материала состава $90 \% \mathrm{BaCO}_{3}+10 \% \mathrm{Y}_{2} \mathrm{O}_{3}$.

Положение края валентной зоны $N_{V S}$ относительно уровня Ферми $E_{\mathrm{F}}$ для первой группы материалов определяли методом ЭСХА с учетом того, что вблизи края валентной зоны плотность электронных состояний $N_{V}(E)$ описывается приближенным соотношением

$$
N_{V}(E) \approx \sqrt{\left|E_{V S}-E\right|}
$$


Таблица 1. Расшифровка $3 d_{5 / 2}$-электронных спектров бария в образцах материалов

\begin{tabular}{|c|c|c|c|c|}
\hline № пика & Энергия пика, eV & Интенсивность пика, counts/s & Ширина пика, eV & Барий в фазе \\
\hline 1 & \multicolumn{4}{|c|}{$90 \% \mathrm{BaCO}_{3}+10 \% \mathrm{Y}_{2} \mathrm{O}_{3}$} \\
\hline 1.1 & 779.70 & 404.39 & 1.40 & $\mathrm{Ba}(\mathrm{OH})_{2} \cdot \mathrm{H}_{2} \mathrm{O}$ \\
\hline 1.2 & 781.15 & 3670.98 & 1.83 & $\mathrm{Ba}_{(1-y-z)} \mathrm{O}_{(1-x)} \mathrm{Ni}_{y} \mathrm{Y}_{z}$ \\
\hline 1.3 & 782.17 & 9302.08 & 1.57 & $\mathrm{BaO}_{(1-x)}$ \\
\hline 1.4 & 782.99 & 3893.82 & 1.34 & $\mathrm{Ba}_{(1-y)} \mathrm{O}_{(1-x)} \mathrm{Y}_{y}$ \\
\hline 2 & \multicolumn{4}{|c|}{$90 \% \mathrm{BaCO}_{3}+10 \% \mathrm{Re}$} \\
\hline 2.1 & 780.32 & 1845.19 & 1.80 & $\mathrm{Ba}_{(1-y-z)} \mathrm{O}_{(1-x)} \mathrm{Ni}_{y} \mathrm{Re}_{z}$ \\
\hline 2.2 & 781.71 & 13073.08 & 1.87 & $\mathrm{BaO}_{(1-x)}$ \\
\hline 2.3 & 782.58 & 609.98 & 0.96 & $\mathrm{Ba}_{(1-y)} \mathrm{O}_{(1-x)} \mathrm{Re}_{y}$ \\
\hline 3 & \multicolumn{4}{|c|}{$90 \% \mathrm{BaCO}_{3}+10 \% \mathrm{Pd}$} \\
\hline 3.1 & 780.64 & 2219.60 & 1.80 & $\mathrm{Ba}_{(1-y-z)} \mathrm{O}_{(1-x)} \mathrm{Ni}_{y} \mathrm{Pd}_{z}$ \\
\hline 3.2 & 781.79 & 12472.40 & 1.88 & $\mathrm{BaO}_{(1-x)}$ \\
\hline 3.3 & 782.61 & 1622.85 & 1.16 & $\mathrm{Ba}_{(1-y)} \mathrm{O}_{(1-x)} \mathrm{Pd}_{y}$ \\
\hline 4 & \multicolumn{4}{|c|}{$90 \% \mathrm{BaCO}_{3}+10 \% \mathrm{SrCO}_{3}$} \\
\hline 4.1 & 780.55 & 1426.20 & 1.99 & $\mathrm{Ba}_{(1-y-z)} \mathrm{O}_{(1-x)} \mathrm{Ni}_{y} \mathrm{Sr}_{z}$ \\
\hline 4.2 & 782.14 & 7593.82 & 1.85 & $\mathrm{BaO}_{(1-x)}$ \\
\hline 4.3 & 783.07 & 1129.03 & 1.04 & $\mathrm{Ba}_{(1-y)} \mathrm{O}_{(1-x)} \mathrm{Sr}_{y}$ \\
\hline 5 & \multicolumn{4}{|c|}{$90 \% \mathrm{~W}+5 \% 2.5 \mathrm{BaO} \cdot 0.4 \mathrm{CaO} \cdot \mathrm{Al}_{2} \mathrm{O}_{3}+5 \% \mathrm{Re}_{2} \mathrm{Sc}$} \\
\hline 5.1 & 779.86 & 478.98 & 1.08 & $\mathrm{Ba}_{(1-y)} \mathrm{O}_{(1-x)} \mathrm{W}_{y}$ \\
\hline 5.2 & 780.84 & 2176.37 & 1.98 & Барий в алюминате бария-кальция \\
\hline 5.3 & 782.30 & 1174.76 & 1.55 & $\mathrm{Ba}_{(1-y)} \mathrm{O}_{(1-x)} \mathrm{Sc}_{y}$ \\
\hline 5.4 & 782.66 & 192.66 & 0.72 & $\mathrm{Ba}_{(1-y)} \mathrm{O}_{(1-x)} \mathrm{Al}_{y}$ \\
\hline 5.5 & 783.63 & 2364.52 & 1.96 & $\mathrm{Ba}_{(1-y-z)} \mathrm{O}_{(1-x)} \mathrm{Re}_{y} \mathrm{Sc}_{z}$ \\
\hline 5.6 & 784.68 & 685.53 & 1.23 & $\mathrm{Ba}_{(1-y)} \mathrm{O}_{(1-x)} \mathrm{Ca}_{y}$ \\
\hline 5.7 & 785.84 & 497.79 & 1.98 & $\mathrm{BaO}_{2}$ \\
\hline 6 & \multicolumn{4}{|c|}{$90 \% \mathrm{~W}+5 \% 2.5 \mathrm{BaO} \cdot 0.4 \mathrm{CaO} \cdot \mathrm{Al}_{2} \mathrm{O}_{3}+5 \%(80 \% \mathrm{~W}+20 \% \mathrm{Re})$} \\
\hline 6.1 & 780.61 & 1978.24 & 1.74 & Барий в алюминате бария-кальция \\
\hline 6.2 & 781.75 & 591.98 & 1.12 & $\mathrm{BaO}_{(1-x)}$ \\
\hline 6.3 & 782.77 & 1656.22 & 1.66 & $\mathrm{Ba}_{(1-y)} \mathrm{O}_{(1-x)} \mathrm{Re}_{y}$ \\
\hline 6.4 & 784.19 & 2550.55 & 1.99 & $\mathrm{Ba}_{(1-y-z)} \mathrm{O}_{(1-x)} \operatorname{Re}_{y} \mathrm{~W}_{z}$ \\
\hline 6.5 & 785.66 & 860.26 & 1.99 & $\mathrm{BaO}_{2}$ \\
\hline 7 & \multicolumn{4}{|c|}{$90 \% \mathrm{~W}+5 \% 2.5 \mathrm{BaO} \cdot 0.4 \mathrm{CaO} \cdot \mathrm{Al}_{2} \mathrm{O}_{3}+5 \% \mathrm{ScH}_{2}$} \\
\hline 7.1 & 780.26 & 1289.40 & 1.45 & Барий в алюминате бария-кальция \\
\hline 7.2 & 781.36 & 2379.82 & 1.98 & $\mathrm{Ba}_{(1-y)} \mathrm{O}_{(1-x)} \mathrm{Sc}_{y}$ \\
\hline 7.3 & 782.46 & 560.63 & 0.99 & $\mathrm{Ba}_{(1-y)} \mathrm{O}_{(1-x)} \mathrm{Al}_{y}$ \\
\hline 7.4 & 783.44 & 1868.78 & 1.99 & $\mathrm{BaCO}_{3}$ \\
\hline 7.5 & 785.09 & 1423.08 & 1.96 & $\mathrm{Ba}_{(1-y-z)} \mathrm{O}_{(1-x)} \mathrm{Sc}_{y} \mathrm{~W}_{z}$ \\
\hline 7.6 & 786.38 & 316.12 & 1.10 & $\mathrm{BaO}_{2}$ \\
\hline
\end{tabular}


Таблица 2. Расшифровка электронного спектра иттрия в материале $90 \% \mathrm{BaCO}_{3}+10 \% \mathrm{Y}_{2} \mathrm{O}_{3}$

\begin{tabular}{c|c|c|c|c}
\hline № пика & Энергия пика, eV & Интенсивность пика, counts/s & Ширина пика, eV & Иттрий в фазе \\
\hline 1 & 157.14 & 24.77 & 1.95 & $3 d_{5 / 2}-\mathrm{Y}_{(2-y)} \mathrm{O}_{(3-x)} \mathrm{Ba}_{y}$ \\
\hline 2 & 158.74 & 103.36 & 1.61 & $3 d_{3 / 2}-\mathrm{Y}_{(2-y)} \mathrm{O}_{(3-x)} \mathrm{Ba}_{y}$ \\
\hline 3 & 159.90 & 223.86 & 1.58 & $3 d_{5 / 2}-\mathrm{Y}_{2} \mathrm{O}_{(3-x)}$ \\
\hline 4 & 161.64 & 133.73 & 1.50 & $3 d_{3 / 2}-\mathrm{Y}_{2} \mathrm{O}_{(3-x)}$
\end{tabular}

Таблица 3. Схема расшифровки пиков характеристических потерь в материале $90 \% \mathrm{BaCO}_{3}+10 \% \mathrm{Y}_{2} \mathrm{O}_{3}$

\begin{tabular}{|c|c|c|c|c|c|c|c|c|c|}
\hline \multirow{3}{*}{ № пика } & \multirow{3}{*}{$\Delta E, \mathrm{eV}$} & \multirow{3}{*}{ Ширина, eV } & \multicolumn{7}{|c|}{ Ряд } \\
\hline & & & \multicolumn{3}{|c|}{$\mathrm{BaO}_{(1-x)}$} & \multicolumn{2}{|c|}{$\mathrm{Ba}_{(1-y)} \mathrm{O}_{(1-x)} \mathrm{Y}_{y}$} & \multicolumn{2}{|c|}{$\mathrm{Ba}_{(1-y-z)} \mathrm{O}_{(1-x)} \mathrm{Ni}_{y} \mathrm{Y}_{z}$} \\
\hline & & & 1 & 2 & 3 & 4 & 5 & 6 & 7 \\
\hline 0 & 0 & 0 & $0 \mathrm{E}_{\mathrm{S} 1}$ & $0 \mathrm{E}_{\mathrm{D} 1}$ & & $0 \mathrm{E}_{\mathrm{S} 2}$ & $0 \mathrm{E}_{\mathrm{D} 2}$ & $0 \mathrm{E}_{\mathrm{S} 3}$ & $0 \mathrm{E}_{\mathrm{D} 3}$ \\
\hline 1 & 2.05 & 1.08 & $1 \mathrm{E}_{\mathrm{S} 1}$ & & & & & & \\
\hline 2 & 3.23 & 1.96 & & $1 \mathrm{E}_{\mathrm{D} 1}$ & $0 \mathrm{E}_{\mathrm{S} 1}+1 \mathrm{E}_{\mathrm{D} 1}$ & & & & \\
\hline 3 & 4.17 & 0.76 & $2 \mathrm{E}_{\mathrm{S} 1}$ & & & & & & \\
\hline 4 & 5.03 & 1.52 & & & $1 \mathrm{E}_{\mathrm{S} 1}+1 \mathrm{E}_{\mathrm{D} 1}$ & & & & \\
\hline 5 & 5.61 & 0.68 & & & & $1 \mathrm{E}_{\mathrm{S} 2}$ & & & \\
\hline 6 & 6.52 & 1.52 & $3 \mathrm{E}_{\mathrm{S} 1}$ & $2 \mathrm{E}_{\mathrm{D} 1}$ & & & & & \\
\hline 7 & 7.67 & 1.55 & & & $2 \mathrm{E}_{\mathrm{S} 1}+1 \mathrm{E}_{\mathrm{D} 1}$ & & $1 \mathrm{E}_{\mathrm{D} 2}$ & $1 \mathrm{E}_{\mathrm{S} 3}$ & \\
\hline 8 & 8.46 & 0.74 & $4 \mathrm{E}_{\mathrm{S} 1}$ & & & & & & \\
\hline 9 & 9.27 & 1.65 & & $3 \mathrm{E}_{\mathrm{D} 1}$ & $3 \mathrm{E}_{\mathrm{S} 1}+1 \mathrm{E}_{\mathrm{D} 1}$ & & & & \\
\hline 10 & 10.00 & 0.88 & $5 \mathrm{E}_{\mathrm{S} 1}$ & & & & & & \\
\hline 11 & 11.01 & 1.62 & & & $4 \mathrm{E}_{\mathrm{S} 1}+1 \mathrm{E}_{\mathrm{D} 1}$ & $2 \mathrm{E}_{\mathrm{S} 2}$ & & & $1 \mathrm{E}_{\mathrm{D} 3}$ \\
\hline 12 & 12.36 & 1.22 & $6 \mathrm{E}_{\mathrm{S} 1}$ & & & & & & \\
\hline 13 & 13.27 & 0.78 & & $4 \mathrm{E}_{\mathrm{D} 1}$ & $5 \mathrm{E}_{\mathrm{S} 1}+1 \mathrm{E}_{\mathrm{D} 1}$ & & & & \\
\hline 14 & 14.39 & 1.49 & $7 \mathrm{E}_{\mathrm{S} 1}$ & & & & & & \\
\hline 15 & 15.65 & 1.18 & & & $6 \mathrm{E}_{\mathrm{S} 1}+1 \mathrm{E}_{\mathrm{D} 1}$ & & $2 \mathrm{E}_{\mathrm{D} 2}$ & $2 \mathrm{E}_{\mathrm{S} 3}$ & \\
\hline 16 & 16.44 & 0.84 & $8 \mathrm{E}_{\mathrm{S} 1}$ & $5 \mathrm{E}_{\mathrm{D} 1}$ & & & & & \\
\hline 17 & 17.41 & 1.89 & & & $7 \mathrm{E}_{\mathrm{S} 1}+1 \mathrm{E}_{\mathrm{D} 1}$ & $3 \mathrm{E}_{\mathrm{S} 2}$ & & & \\
\hline 18 & 19.20 & 1.31 & $9 \mathrm{E}_{\mathrm{S} 1}$ & $6 \mathrm{E}_{\mathrm{D} 1}$ & $8 \mathrm{E}_{\mathrm{S} 1}+1 \mathrm{E}_{\mathrm{D} 1}$ & & & & \\
\hline 19 & 20.93 & 1.99 & $10 \mathrm{E}_{\mathrm{S} 1}$ & & & & & & \\
\hline 20 & 21.78 & 0.67 & & & $9 \mathrm{E}_{\mathrm{S} 1}+1 \mathrm{E}_{\mathrm{D} 1}$ & & & & $2 \mathrm{E}_{\mathrm{D} 3}$ \\
\hline 21 & 22.84 & 1.69 & $11 \mathrm{E}_{\mathrm{S} 1}$ & $7 \mathrm{E}_{\mathrm{D} 1}$ & & $4 \mathrm{E}_{\mathrm{S} 2}$ & $3 \mathrm{E}_{\mathrm{D} 2}$ & $3 \mathrm{E}_{\mathrm{S} 3}$ & \\
\hline 22 & 23.91 & 0.72 & & & $10 \mathrm{E}_{\mathrm{S} 1}+1 \mathrm{E}_{\mathrm{D} 1}$ & & & & \\
\hline 23 & 24.58 & 1.42 & $12 \mathrm{E}_{\mathrm{S} 1}$ & & & & & & \\
\hline 24 & 26.54 & 0.91 & $13 \mathrm{E}_{\mathrm{S} 1}$ & $8 \mathrm{E}_{\mathrm{D} 1}$ & $11 \mathrm{E}_{\mathrm{S} 1}+1 \mathrm{E}_{\mathrm{D} 1}$ & & & & \\
\hline 25 & 27.86 & 0.61 & & & $12 \mathrm{E}_{\mathrm{S} 1}+1 \mathrm{E}_{\mathrm{D} 1}$ & $5 \mathrm{E}_{\mathrm{S} 2}$ & & & \\
\hline 26 & 29.40 & 0.44 & $14 \mathrm{E}_{\mathrm{S} 1}$ & $9 \mathrm{E}_{\mathrm{D} 1}$ & $13 \mathrm{E}_{\mathrm{S} 1}+1 \mathrm{E}_{\mathrm{D} 1}$ & & & & \\
\hline
\end{tabular}


Таблица 4. Идентификация рядов характеристических потерь в материале $90 \% \mathrm{BaCO}_{3}+10 \% \mathrm{Y}_{2} \mathrm{O}_{3}$

\begin{tabular}{c|c|c|c|c|c}
\hline № ряда & Уравнение ряда & $E_{S}, \mathrm{eV}$ & $E_{D}, \mathrm{eV}$ & $\bar{E}_{S}, \mathrm{eV}$ & $\bar{E}_{D}, \mathrm{eV}$ \\
\cline { 1 - 4 } 1 & $\Delta E=E_{S .1} \cdot n_{2}$ & 2.10 & - & & \\
\cline { 1 - 4 } 2 & $\Delta E=E_{D .1} \cdot n_{1}$ & - & 3.27 & \multirow{2}{*}{2.06} & \multirow{2}{*}{3.36} \\
\cline { 1 - 4 } 3 & $\Delta E=E_{S .1} \cdot n_{2}+E_{D .1}$ & 2.01 & 3.45 & & \\
\cline { 1 - 4 } 4 & $\Delta E=E_{S .2} \cdot n_{2}$ & 5.57 & - & \multirow{2}{*}{5.57} & \multirow{2}{*}{7.61} \\
\cline { 1 - 4 } 5 & $\Delta E=E_{D .2} \cdot n_{1}$ & - & 7.61 & & \\
\cline { 1 - 4 } 6 & $\Delta E=E_{S .3} \cdot n_{2}$ & 7.61 & - & \multirow{2}{*}{7.61} & \multirow{2}{*}{10.89} \\
\cline { 1 - 4 } 7 & $\Delta E=E_{D .3} \cdot n_{1}$ & - & 10.89 & &
\end{tabular}

Примечание. Индексы „S“ и „D“ относятся к поверхностным и объемным параметрам соответственно. В двух правых столбцах даны усредненные значения.

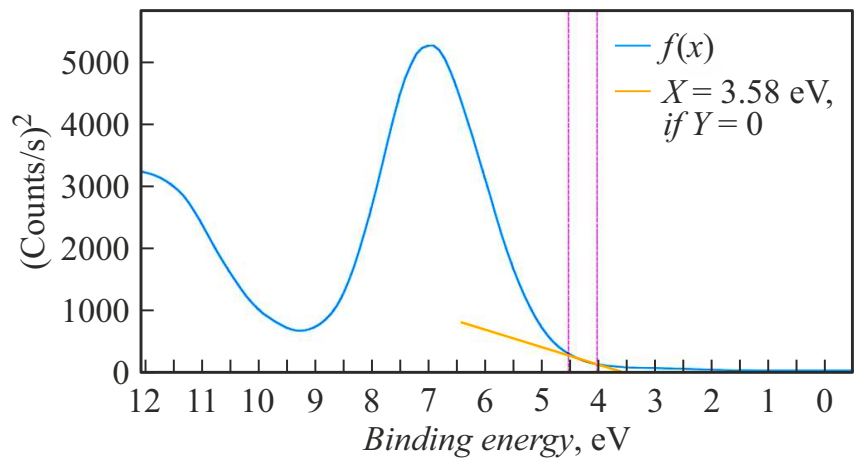

Pис. 5. Структура края валентной зоны образца материала $\mathrm{BaCO}_{3}+10 \% \mathrm{Y}_{2} \mathrm{O}_{3}$, отожженного в вакууме при $1200^{\circ} \mathrm{C}$ в течение $2 \mathrm{~h}$ в координатах „квадрат интенсивности сигнала-энергия связи“.

В этом случае зависимость квадрата интенсивности сигнала ЭСХА от энергии связи будет прямой линией, экстраполяция которой к оси энергии позволяет определить параметр $E_{V S}$ относительной уровня Ферми, как в качестве примера показано на рис. 5 для образца материала состава $90 \% \mathrm{BaCO}_{3}+10 \% \mathrm{Y}_{2} \mathrm{O}_{3}$. Значения параметра $E_{V S}$, в свою очередь, позволяют определить и величину, и направление искривления энергетических зон $V$ в кристаллитах оксида бария у поверхности [14]. Определить параметр $E_{V S}$ для второй группы образцов материалов не представлялось возможным, так как в этих образцах валентная зона кристаллитов оксида бария перекрывается зоной проводимости вольфрама.

\section{2. Экспериментальные результаты и их обсуждение}

В табл. 1 приведены сводные результаты расшифровки химических состояний бария в исследованных образцах.
Положение пиков бария в фазах $\mathrm{Ba}(\mathrm{OH})_{2} \cdot \mathrm{H}_{2} \mathrm{O}$, $\mathrm{BaO}_{(1-x)}, \quad \mathrm{Ba}_{(1-y)} \mathrm{O}_{(1-x)} \mathrm{W}_{y}, \quad 2.5 \mathrm{BaO} \cdot 0.4 \mathrm{CaO} \cdot \mathrm{Al}_{2} \mathrm{O}_{3}$, $\mathrm{Ba}_{(1-y)} \mathrm{O}_{(1-x)} \mathrm{Ca}_{y}, \quad \mathrm{BaCO}_{3}, \quad \mathrm{Ba}_{(1-y)} \mathrm{O}_{(1-x)} \mathrm{Al}_{y}$, $\mathrm{Ba}_{(1-y)} \mathrm{O}_{(1-x)} \mathrm{Sc}_{y}, \quad \mathrm{BaO}_{2}$ хорошо согласуется с данными, приведенными в [14]. Это позволило расшифровать и пики состояний бария в кристаллитах $\mathrm{BaO}$, легированных иттрием, рением, палладием, стронцием, в том числе при легировании двумя типами атомов. Полученные результаты подтверждают высказанное выше предположение о том, что в процессе формирования в исследованных образцах материалов эмиссионно-активных кристаллитов $\mathrm{BaO}$, содержащих кислородные вакансии, формируются и кристаллиты $\mathrm{BaO}$, легированные одним или двумя типами микропримесей элементов их состава исходных фаз.

Из приведенной в качестве примера табл. 2 видно, что в образце материала $90 \% \mathrm{BaCO}_{3}+10 \% \mathrm{Y}_{2} \mathrm{O}_{3}$ в процессе прогрева в вакууме параллельно формируются и кристаллиты оксида иттрия, содержащие кислородные вакансии, а также кристаллиты оксида иттрия, содержащие кислородные вакансии и микропримеси атомов бария. Данный результат важен для понимания физикохимических процессов, протекающих в других типах катодных материалов - высокотемпературных катодов на основе оксида иттрия и тантала (синтерированные катоды) и оксида иттрия, оксида алюминия и вольфрама (металлокерамические катоды), которые нашли применение в мощных СВЧ магнетронах.

Как видно из перечня характеристических потерь энергии электронов в образце материала $90 \% \mathrm{BaCO}_{3}+10 \% \mathrm{Y}_{2} \mathrm{O}_{3}$ (табл. 3), все пики могут быть сгруппированы в несколько рядов, которые описываются уравнением (2). Указанные ряды, приведенные в качестве примера для материала $90 \% \mathrm{BaCO}_{3}+10 \% \mathrm{Y}_{2} \mathrm{O}_{3}$ в табл. 4 , позволяют определить энергии поверхностных и объемных плазмонов и вычислить величины объемной и поверхностной концентраций кислородных вакансий для оксидных фаз и величины объемной и поверхностной электронной концентрации для металлических фаз. Аналогичные результаты были получены и для других исследованных материалов. В табл. 5 приведены сводные данные указанным параметрам электронной структуры фаз, сформировавшихся в составе исследованных образцов материалов в результате их отжига в вакууме. При расшифровке фаз в табл. 5 учитывали результаты табл. 1, а также принимали во внимание значение интенсивностей пиков характеристических потерь, приведенных на рис. 6 в качестве примера для материала $90 \% \mathrm{BaCO}_{3}+10 \% \mathrm{Y}_{2} \mathrm{O}_{3}$. В табл. 5 жирным шрифтом в правом столбце выделены фазы, для которых имеют место минимальные значения отношения поверхностной и объемной концентрации кислородных вакансий.

Для определения влияния фазового состояния скандия (в составе оксида, интерметаллида, гидрида) в катодном материале на процесс формирования кислородных вакансий в кристаллитах $\mathrm{BaO}$, на рис. 6 приведены концентрационные зависимости (от содержания оксида 
Таблица 5. Параметры характеристических потерь $\left(E_{S}, E_{D}\right)$, а также концентрации кислородных вакансий $\left(N_{S}, N_{D}\right)$ в катодных материалах

\begin{tabular}{|c|c|c|c|c|c|c|}
\hline \multirow{2}{*}{ № фазы } & \multirow{2}{*}{ Фаза } & \multicolumn{5}{|c|}{ Параметр } \\
\hline & & $E_{S}, \mathrm{eV}$ & $E_{D}, \mathrm{eV}$ & $N_{S}, \mathrm{~m}^{-3}$ & $N_{D}, \mathrm{~m}^{-3}$ & $N_{S} / N_{D}$ \\
\hline 1 & \multicolumn{6}{|c|}{$90 \% \mathrm{BaCO}_{3}+10 \% \mathrm{Y}_{2} \mathrm{O}_{3}$} \\
\hline 1.1 & $\mathrm{Ba}_{(1-y)} \mathrm{O}_{(1-x)} \mathrm{Y}_{y}$ & 2.06 & 3.36 & $5.38 \cdot 10^{24}$ & $7.15 \cdot 10^{24}$ & 0.75 \\
\hline 1.2 & $\mathrm{BaO}_{(1-x)}$ & 5.57 & 7.61 & $4.45 \cdot 10^{25}$ & $4.15 \cdot 10^{25}$ & 1.07 \\
\hline 1.3 & $\mathrm{Ba}_{(1-y-z)} \mathrm{O}_{(1-x)} \mathrm{Ni}_{y} \mathrm{Y}_{z}$ & 7.61 & 10.89 & $4.53 \cdot 10^{25}$ & $4.63 \cdot 10^{25}$ & 0.98 \\
\hline 2 & \multicolumn{6}{|c|}{$90 \% \mathrm{BaCO}_{3}+10 \% \mathrm{Re}$} \\
\hline 2.1 & $\mathrm{BaO}_{(1-x)}$ & 2.23 & 2.89 & $6.30 \cdot 10^{24}$ & $5.29 \cdot 10^{24}$ & 1.19 \\
\hline 2.2 & $\mathrm{Ba}_{(1-y)} \mathrm{O}_{(1-x)} \mathrm{Re}_{y}$ & 3.78 & 5.03 & $1.55 \cdot 10^{25}$ & $1.37 \cdot 10^{25}$ & 1.13 \\
\hline 2.3 & $\mathrm{Ba}_{(1-y-z)} \mathrm{O}_{(1-x)} \mathrm{Ni}_{y} \mathrm{Re}_{z}$ & 7.21 & 10.05 & $4.06 \cdot 10^{25}$ & $3.95 \cdot 10^{25}$ & 1.03 \\
\hline 3 & \multicolumn{6}{|c|}{$90 \% \mathrm{BaCO}_{3}+10 \% \mathrm{Pd}$} \\
\hline 3.1 & $\mathrm{BaO}_{(1-x)}$ & 2.43 & 3.08 & $7.48 \cdot 10^{24}$ & $6.01 \cdot 10^{24}$ & 1.25 \\
\hline 3.2 & $\mathrm{Ba}_{(1-y)} \mathrm{O}_{(1-x)} \mathrm{Pd}_{y}$ & 3.83 & 5.49 & $1.26 \cdot 10^{25}$ & $1.33 \cdot 10^{25}$ & 0.95 \\
\hline 3.3 & $\mathrm{Ba}_{(1-y-z)} \mathrm{O}_{(1-x)} \mathrm{Ni}_{y} \mathrm{Pd}_{z}$ & 7.36 & 10.62 & $4.23 \cdot 10^{25}$ & $4.41 \cdot 10^{25}$ & 0.96 \\
\hline 4 & \multicolumn{6}{|c|}{$90 \% \mathrm{BaCO}_{3}+10 \% \mathrm{SrCO}_{3}$} \\
\hline 4.1 & $\mathrm{BaO}_{(1-x)}$ & 2.20 & 2.90 & $6.13 \cdot 10^{24}$ & $5.33 \cdot 10^{24}$ & 1.15 \\
\hline 4.2 & $\mathrm{Ba}_{(1-y-z)} \mathrm{O}_{(1-x)} \mathrm{Ni}_{y} \mathrm{Sr}_{z}$ & 3.82 & 5.79 & $1.72 \cdot 10^{25}$ & $1.97 \cdot 10^{25}$ & 0.87 \\
\hline 4.3 & $\mathrm{Ba}_{(1-y)} \mathrm{O}_{(1-x)} \mathrm{Sr}_{y}$ & 7.78 & 11.29 & $4.73 \cdot 10^{25}$ & $4.98 \cdot 10^{25}$ & 0.94 \\
\hline 5 & \multicolumn{6}{|c|}{$90 \% \mathrm{~W}+5 \% 2.5 \mathrm{BaO} \cdot 0.4 \mathrm{CaO} \cdot \mathrm{Al}_{2} \mathrm{O}_{3}+5 \% \mathrm{Re}_{2} \mathrm{Sc}$} \\
\hline 5.1 & $\mathrm{Ba}_{(1-y)} \mathrm{O}_{(1-x)} \mathrm{Sc}_{y}$ & 2.22 & 3.41 & $6.25 \cdot 10^{24}$ & $7.37 \cdot 10^{24}$ & 0.85 \\
\hline 5.2 & $\mathrm{Ba}_{(1-y)} \mathrm{O}_{(1-x)} \mathrm{W}_{y}$ & 8.61 & 11.72 & $1.32 \cdot 10^{26}$ & $1.22 \cdot 10^{26}$ & 1.08 \\
\hline 5.3 & $\mathrm{Ba}_{(1-y-z)} \mathrm{O}_{(1-x)} \operatorname{Re}_{y} \mathrm{Sc}_{z}$ & 8.80 & 12.24 & $8.41 \cdot 10^{25}$ & $8.14 \cdot 10^{25}$ & 1.03 \\
\hline 5.4 & $\mathrm{~W}$ & 7.82 & 11.26 & $8.89 \cdot 10^{28}$ & $9.21 \cdot 10^{28}$ & 0.97 \\
\hline 6 & \multicolumn{6}{|c|}{$90 \% \mathrm{~W}+5 \% 2.5 \mathrm{BaO} \cdot 0.4 \mathrm{CaO} \cdot \mathrm{Al}_{2} \mathrm{O}_{3}+5 \%(80 \% \mathrm{~W}+20 \% \mathrm{Re})$} \\
\hline 6.1 & $\mathrm{BaO}_{(1-x)}$ & 2.35 & 3.23 & $7.00 \cdot 10^{24}$ & $6.61 \cdot 10^{24}$ & 1.06 \\
\hline 6.2 & $\mathrm{Ba}_{(1-y-z)} \mathrm{O}_{(1-x)} \operatorname{Re}_{y} \mathrm{~W}_{z}$ & 5.81 & 8.85 & $3.67 \cdot 10^{25}$ & $4.26 \cdot 10^{25}$ & 0.86 \\
\hline 6.3 & $\mathrm{Ba}_{(1-y)} \mathrm{O}_{(1-x)} \mathrm{Re}_{y}$ & 9.70 & 13.50 & $1.68 \cdot 10^{26}$ & $1.63 \cdot 10^{26}$ & 1.03 \\
\hline 6.4 & $\mathrm{~W}$ & 7.86 & 10.94 & $8.98 \cdot 10^{28}$ & $8.70 \cdot 10^{28}$ & 0.72 \\
\hline 7 & \multicolumn{6}{|c|}{$90 \% \mathrm{~W}+5 \% 2.5 \mathrm{BaO} \cdot 0.4 \mathrm{CaO} \cdot \mathrm{Al}_{2} \mathrm{O}_{3}+5 \% \mathrm{ScH}_{2}$} \\
\hline 7.1 & $\mathrm{Ba}_{(1-y)} \mathrm{O}_{(1-x)} \mathrm{Sc}_{y}$ & 2.15 & 3.58 & $5.86 \cdot 10^{24}$ & $8.12 \cdot 10^{24}$ & 0.72 \\
\hline 7.2 & $\mathrm{Ba}_{(1-y-z)} \mathrm{O}_{(1-x)} \mathrm{Sc}_{y} \mathrm{~W}_{z}$ & 5.40 & 8.09 & $1.86 \cdot 10^{25}$ & $2.09 \cdot 10^{25}$ & 0.89 \\
\hline 7.3 & $\mathrm{~W}$ & 7.27 & 10.23 & $7.68 \cdot 10^{28}$ & $7.60 \cdot 10^{28}$ & 1.01 \\
\hline
\end{tabular}

Пр и ме чани е. Индексы „,“ и „ $D^{“}$ относятся к поверхностным и объемным параметрам соответственно.

скандия) объемной и поверхностной концентраций кислородных вакансий в образцах материалов на основе порошков вольфрама и фазы $2.5 \mathrm{BaO} \cdot 0.4 \mathrm{CaO} \cdot \mathrm{Al}_{2} \mathrm{O}_{3}$, в которой оксид алюминия полностью или частично заменялся на оксид скандия [14]. Из рис. 6 видно, что в широком интервале содержания оксида скандия отношение поверхностной и объемной концентрации вакансий остается неизменным и равным примерно 1.1, что контрастирует с выделенными ячейками в правом столбце табл. 5. 
Таблица 6. Параметры искривления энергетических зон у поверхности кристаллитов оксида бария (V)

\begin{tabular}{c|c|c|c|c|c|c}
\hline \multirow{2}{*}{ Параметр } & \multicolumn{5}{|c}{ Образец материала } \\
\cline { 2 - 7 } & $\mathrm{BaCO}_{3}[9]$ & $\mathrm{BaCO}_{3}+\mathrm{CaCO}_{3}[9]$ & $\mathrm{BaCO}_{3}+\mathrm{Y}_{2} \mathrm{O}_{3}$ & $\mathrm{BaCO}_{3}+\mathrm{Re}$ & $\mathrm{BaCO}_{3}+\mathrm{Pd}^{2}$ & $\mathrm{BaCO}_{3}+\mathrm{SrCO}_{3}$ \\
\hline$E_{V S}, \mathrm{eV}$ & 2.70 & 2.75 & $\mathbf{4 . 1 5}$ & 3.45 & $\mathbf{3 . 8 3}$ & 3.48 \\
$V, \mathrm{eV}$ & 1.33 & 1.28 & $-\mathbf{0 . 1 2}$ & 0.58 & $\mathbf{0 . 2 0}$ & 0.55
\end{tabular}

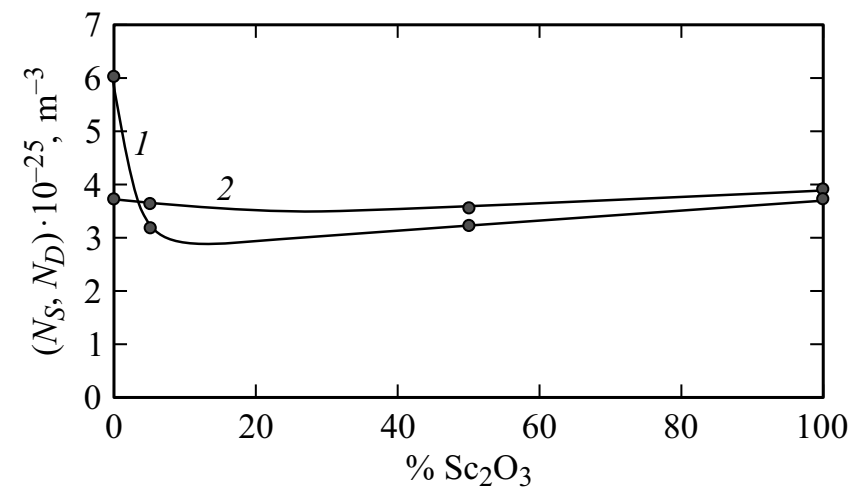

Рис. 6. Зависимости объемной (1) и поверхностной (2) концентраций кислородных вакансий от содержания $\mathrm{Sc}_{2} \mathrm{O}_{3}$ в катодных материалах.

При изучении положения верхнего края валентной зоны в оксидных фазах методом ЭСХА, как показано на рис. 5, отсутствует возможность раздельного определения положения края валентной зоны для каждой оксидной фазы по отдельности в случае, когда имеется несколько оксидных фаз. В то же время суммарное положение верха валентной зоны и определяет величину суммарного искривления энергетических зон, т.е. величину работы выхода материала. В табл. 6 приведены значения положения верха валентной зоны относительно уровня Ферми $\left(E_{V S}\right)$ и величины суммарного искривления энергетических зон $(V)$ кристаллитов оксида бария для исследованных материалов. Оптимальные варианты микролегирования $\mathrm{BaO}$ выделены жирным шрифтом.

При отжиге карбоната бария или алюмината бариякальция в вакууме в присутствии других фаз установившаяся объемная концентрация кислородных вакансий в кристаллитах $\mathrm{BaO}$ зависит не только от исходного фазового состава материала, но и от температуры и времени отжига, пористости материала и гранулометрического состава порошковых компонентов. Собственно, в оптимизации гранулометрического состава, пористости, температуры и времени отжига и состоит суть активирования катодного материала, в результате которого в объеме кристаллитов $\mathrm{BaO}$ формируется требуемая концентрация кислородных вакансий. Однако верхний монослой кристаллитов $\mathrm{BaO}$ в каждый момент времени находит- ся в термодинамическом равновесии с объемом $\mathrm{BaO}$, при этом

- поверхностная концентрация вакансий, как впервые показано в [13] и видно из результатов настоящей работы, определяется не только равновесием между объемом и поверхностью кристаллита, но и наличием легирующих (примесных) атомов в верхнем монослое кристаллита;

- в свою очередь, именно поверхностная концентрация кислородных вакансий определяет величину искривления энергетических зон у поверхности кристаллитов оксида бария: чем меньше величина искривления, тем меньше и величина работы выхода [13].

В работе [13] была предложена теория скандатных катодов, согласно которой низкую работу выхода кристаллита оксида бария можно обеспечить путем формирования нанокристаллита $\mathrm{BaO}$, в верхнем монослое которого атомы бария полностью или частично заменены атомами скандия. Признаком формирования такой структуры является снижение поверхностной концентрации кислородных вакансий по сравнению с объемной концентрацией кислородных вакансий. Условием формирования такой структуры является размерный фактор легирующего элемента - его ионный радиус должен быть примерно равен 0.60 от ионного радиуса бария. Под данный критерий попадает и никель, ионный радиус которого составляет 0.54 от ионного радиуса бария. На основе теоретического подхода [13] можно сформулировать и более общие условия, обеспечивающие снижение поверхностной концентрации кислородных вакансий в кристаллитах оксида бария относительно ее объемной концентрации и являющиеся обобщением работы [13] и экспериментальных результатов настоящей работы:

- энтальпия образования оксида легирующего элемента должна быть выше энтальпии образования оксида бария, т.е. энергия связи „ион кислорода-ион легирующего элемента“ должна быть выше энергии связи „ион кислорода-ион бария“;

- для эффективного легирования кристаллитов оксида бария другим элементом, этот элемент в исходной фазе должен находиться в слабосвязанной форме, например, в составе интерметаллида, гидрида или нанокристаллического оксида с высокой избыточной поверхностной энергией;

- для сегрегации легирующего элемента именно в верхнем монослое кристаллита оксида бария указанный 
кристаллит должен находиться в виде нанокристаллов, в которых имеет место значительное различие межплоскостного расстояния между первым и вторым монослоями и монослоями в объеме нанокристаллита. В этом случае и вступает в действие размерный фактор легирующего элемента.

Именно указанные условия, как видно из табл. 5, выполняются при введении в исходный карбонат бария дополнительных компонентов в виде интерметаллида $\mathrm{Re}_{2} \mathrm{Sc}$ и гидрида $\mathrm{ScH}_{2}$, выполняются при малом содержании дополнительного компонента в виде оксида скандия и нарушаются при высокой концентрации оксида скандия в материале. Эти же условия выполняются и при совместном легировании оксида бария никелем и стронцием, на чем, собственно, и основаны высокие термоэмиссионные свойства оксидно-никелевых катодов.

\section{Заключение}

1. Критериями достижения минимума работы выхода катодного материала с кристаллитами $\mathrm{BaO}$ в качестве эмиссионно-активного компонента являются обеспечение минимума отношения поверхностной и объемной концентрации кислородных вакансий в $\mathrm{BaO}$ и максимума расстояния между верхом валентной зоны и уровнем Ферми с $\mathrm{BaO}$.

2. Высокие термоэмиссионные свойства скандатного катода могут быть обеспечены путем формирования в катодном материале кристаллитов оксида бария, в которых в верхнем монослое атомы бария полностью или частично заменены на атомы скандия.

3. Обеспечить эффективное легирование кристаллитов атомами скандия возможно при использовании в составе катодного материала скандия в составе интерметаллида скандия, гидрида скандия или оксида скандия в нанокристаллическом состоянии.

4. Обеспечить сегрегацию атомов скандия именно в верхнем монослое кристаллитов оксида бария возможно путем формирования указанных кристаллитов на этапе активирования катода в виде нанокристаллитов оксида бария.

5. Формирование именно нанокристаллитов оксида бария на этапе активирования катода возможно путем использования активатора разложения алюмината бариякальция (вольфрама) в виде нанокристаллов вольфрама или паров низших оксидов вольфрама, поступающего к поверхности алюмината из объема катода по порам в алюминате.

\section{Конфликт интересов}

Авторы заявляют, что у них нет конфликта интересов.

\section{Список литературы}

[1] G. Gartner, P. Geintter, A. Ritz. Appl. Surf. Sci., 111, 11 (1997).

[2] I.I. Bekh, O.I. Getman, V.V. Il'chenko, A.E. Lushkin, V.V. Panichkina, S.P. Rakitin. Ukr. J. Phys., 54 (3), 297 (2009).

[3] I. Brodie, B. Vancil. Proc. IEEE Int. Vacuum Electron. Conf., Monterey, CA, United States. 2014. P. 53-54.

[4] C. Lai, J.S. Wang, F. Zhou, W. Liu, P. Hu, C.H. Wang, R.Z. Wang, N.H. Miao. Appl. Surf. Sci., 440, 763 (2018).

[5] X. Liu, B. Vancil, M. Beck, T. Balk. Materials, 12, 636 (2019).

[6] F. Yang, J. Wang, W. Liu, Y. Wang, M. Zhou. Proc. IEEE Int. Vacuum Electron. Conf., Monterey, CA, United States. 2014. P. 55-56.

[7] В.И. Капустин. Известия АН СССР, сер. физ., 55 (12), 2455 (1991).

[8] В.И. Капустин. Перспективные материалы, 2, 5 (2000).

[9] В.И. Капустин, И.П. Ли, В.С. Петров, Н.Е. Леденцова, А.В. Турбина. Электронная техника, сер. 1: СВЧ-техника, 1 (528), 8 (2016).

[10] И.П. Ли, В.С. Петров, Т.В. Прокофьева, Н.Е. Леденцова, А.В. Шуманов, А.Д. Силаев, В.С. Поляков, В.И. Капустин, В.И. Свитов. Электронная техника, сер. 1: СВЧ-техника, 2 (525), 45 (2015).

[11] В.И. Капустин, И.П. Ли, А.В. Шуманов, Ю.Ю. Лебединский, А.В. Заблоцкий. ЖТФ, 87 (1), 105 (2017). [V.I. Kapustin, I.P. Li, A.V. Shumanov, Yu.Yu. Lebedinskii, A.V. Zablotskii. Tech. Phys., 62 (1), 116 (2017).]

[12] В.И. Капустин, И.П. Ли, А.В. Шуманов, С.О. Москаленко, В.И. Свитов. Перспективные материалы, 3, 19 (2019). [V.I. Kapustin, I.P. Li, A.V. Shumanov, S.O. Moskalenko, V.I. Svitov. Inorganic Mater.: Appl. Res., 10 (5), 1065 (2019).]

[13] В.И. Капустин, И.П. Ли, А.В. Шуманов, С.О. Москаленко. ЖТФ, 90 (1), 161 (2020). [V.I. Kapustin, I.P. Li, A.V. Shumanov, S.O. Moskalenko. Tech. Phys., 65 (1), 151 (2020).]

[14] В.И. Капустин, И.П. Ли. Теория, электронная структура и физикохимия материалов катодов СВЧ приборов (ИНФРА-М, М., 2020) 